\title{
Modified MIMO Cube for Enhanced Channel Capacity
}

\author{
Lajos Nagy \\ Department of Broadband Infocommunications and Electromagnetic Theory, Budapest University of Technology and Economics, \\ Goldmann György ter 3, 1111 Budapest, Hungary
}

Correspondence should be addressed to Lajos Nagy, nagy@mht.bme.hu

Received 1 August 2011; Revised 9 October 2011; Accepted 18 October 2011

Academic Editor: Wenhua Chen

Copyright () 2012 Lajos Nagy. This is an open access article distributed under the Creative Commons Attribution License, which permits unrestricted use, distribution, and reproduction in any medium, provided the original work is properly cited.

This paper deals with the optimization of MIMO antenna elements' position in modified MIMO cube for getting maximal channel capacity in indoor environment. The dependence of the channel capacity on the antenna orientation was analyzed by simulations. We have also examined the effect of the frequency dependence of the antenna system (in case of conjugate matching and nonconjugate matching) for the channel capacity. Based on the simulation results in the created and measured antenna system, the antennas were at a right angle to each other. At the two chosen different structures, we measured the antenna parameters and the channel capacity. In this paper, we present the results of the measurements which clearly confirm our simulations. We will point out the differences between the two antenna structures.

\section{Introduction}

Wideband indoor wireless systems are gaining increasing importance nowadays. This is why the analysis of MIMO systems which eliminate the problems of indoor propagation is of primary significance. In case of indoor propagation, a frequent problem is that there are disturbing objects between the transmitter and the receiver antennas consequently there is no direct line of sight in the wireless channel. The objects in the channel adversely affect the transmission because they scatter and reflect the signals, resulting in attenuation and phase errors. MIMO systems can be a solution to these problems.

MIMO system can eliminate the phase, distance, and polarization diversity. Thus, in an indoor environment, the theoretically highest channel capacity can be nearly achieved. It is known that the channel capacity scales linearly with the number of antennas at both the receiver and transmitter for complex Gaussian fading channels.

When designing a complete multiple-antenna system, we have to try to approach a maximal mean capacity with a minimal number of antennas in the system. For multipleantenna systems, an important problem is the reduction of the number of antennas for practicability and usability reasons. We will assume that the multiple-antenna system with three elements on both the receiver and transmitter issues is the simplest structure for the highest mean capacity.

When finding the best totally free structure for optimal channel capacity, the simulations come too little if the analyzed structures are chosen randomly and because most of the structure would be analyzed. Therefore, we have searched a right method for the optimal selection of the structure.

The MIMO cube as a compact antenna is one of a possible realization for MIMO antenna system [1, 2], but especially for higher frequencies the loss and coupling between the dipoles decreases the capacity of the system. The slot dipole is promising a cheap and lossless solution for MIMO cube, and this was investigated also in form of simulations and measurements. In [3] the MIMO antenna formed by slot antennas is already presented by our group, and later in many researches slot antennas are used as MIMO elementary antennas [4-7]. In this paper, a MIMO cube prototype consisting of three slot-type dipole antennas is fabricated and tested accordingly.

The authors of [8] reported study on the effect of mutual coupling. In [9] the effect of mutual coupling has been shown to reduce capacity substantially especially when packing higher number of receiving antennas for a fixed aperture. Based on this published effect, our present investigation 
takes into account the mutual coupling at the capacity optimization procedure at the MIMO antenna design.

The MIMO capacity optimization procedure uses the (Genetic Algorithm) GA which has its main advantages, cannot stick in a local extreme, can be simply implemented, and can find the global minimum or maximum of the composite multivariable function. Like the terrestrial evolution, the GA handles the functional parameters as biological gene. The different input parameters are crossed among the population where the population is defined as a set of all-time actual available input parameters. Just like in biology, the survival rate of the strongest candidates has a higher probability; furthermore, the reproduction of the next-generation parameter set is influenced by mutation of earlier analyzed-selected input parameters. The variety of the first-generation initial input parameter set is guaranteed by initialization of the GA with random values [10-12]. The antenna orientations are the optimized variables in our GA procedures, and the cost function is the channel capacity.

The scattering environment model which we applied in the simulation was a three-dimensional (3D) doublebouncing (DB) stochastic scattering channel model with wide angular spread as a basis at both ends. By using this model, we wrote down perfectly an unmitigated office indoor environment.

In this model, the scatterers are on a spherical surface around transmitter and receiver antennas. In an indoor scenario, the standard propagation is NLOS (nonline of sight); thus, there are several jamming objects between the transmitter and the receiver devices. The disturbing objects around the antennas change phase and level of transmitted signal. The model statistically describes the material, surface, and place of these objects which results in phase and amplitude error in the course of propagation. Waves of arbitrary polarizations are incident on the antenna structure from all possible directions as can be seen in Figure 12. The transmission matrix $(\mathbf{H})$ which connects the receiver and transmitter antennas is filled by assuming DB scattering. The effect of mutual coupling is taken into account in the channel matrixes by using mutual impedances. By this method, we could describe the continuously varying indoor environment.

In this paper, finally, we present simulation and measurement results for the channel capacity of a $3 \times 3 \mathrm{MIMO}$ antenna system. The aim of this research is the enhancing of the MIMO channel capacity for indoor environment.

In addition we have examined the effect of the frequency dependence of the antenna system (in case of conjugate matching and nonconjugate matching) for the channel capacity. Based on the result of the simulation, we have created the antenna system and measured the antenna parameters and the channel capacity. In this paper, we would like to show the results of the measurements which clearly confirm our simulations. We will point out the differences which are between the two antenna structures and certificate our allegations.

At the research work, the target frequency for the MIMO antennas were different for Sections 2 and 5. On the one hand, the working frequency for first experimental version

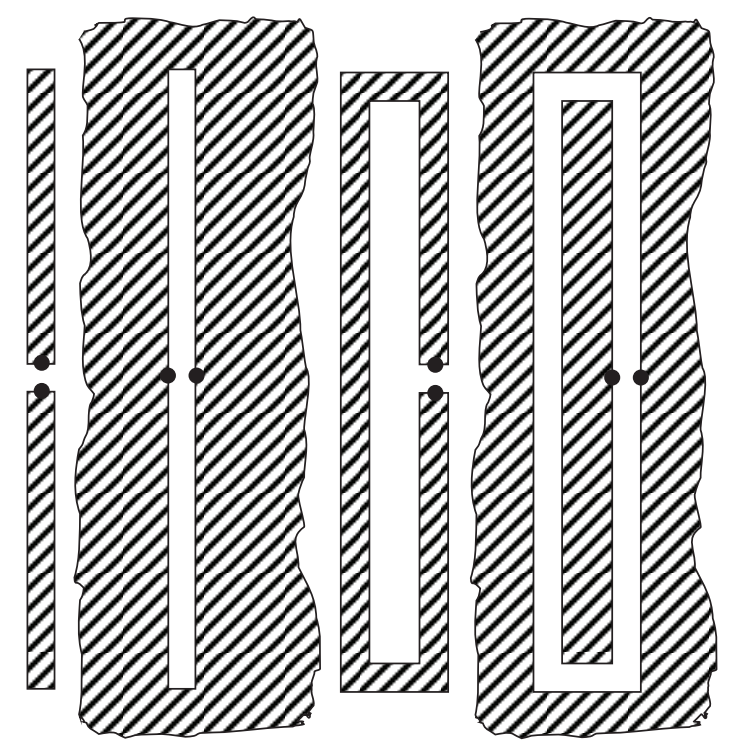

Figure 1: Dipole and folded dipole as complementary structures.

was at $4.6 \mathrm{GHz}$ for a special requirement, on the other hand the channel capacity enhancement and MIMO antenna design was performed at $2.2 \mathrm{GHz}$ range as the possible UMTS application.

\section{The MIMO Antenna}

Slot dipole antennas are used as basic elements of the MIMO antennas investigated. Experimental results show that especially at higher frequencies of various wireless applications the additional loss of the printed antennas increase, and therefore implementation of such antennas is not always possible above 1-2 GHz.

In our first test the single-slot antennas were designed, manufactured, and measured without taking into account the mutual coupling between them in case of forming MIMO antennas from them.

Wire dipole and a slot are complementary antennas. The solution for the slot can be found from the solution to an equivalent dipole by an interchange of the electric and magnetic fields. Not only the pattern but also the input impedance can be found.

Figure 1 shows two such complementary structures. Babinet's principle of optical screens extended by Booker is used to vector electromagnetic fields. If we take two such complementary screens and perform line integrals over identical paths to compute the impedance of each, we obtain the result for the input impedances

$$
Z_{1} \cdot Z_{c}=\frac{\eta^{2}}{2}
$$

where $Z_{1}$ is the input impedance of the structure, $Z_{c}$ the input impedance of the complementary structure, and $\eta$ the impedance of free space $(120 \pi)$.

Both the structure and its complementary one radiate the same pattern but differ in polarization. 

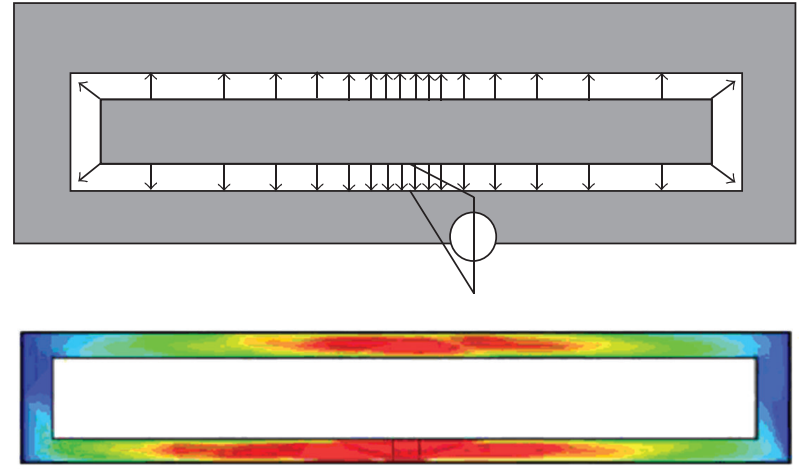

Figure 2: Electrical field strength on the slot, theoretical and simulated.

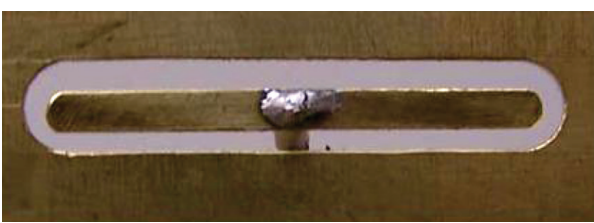

(a)

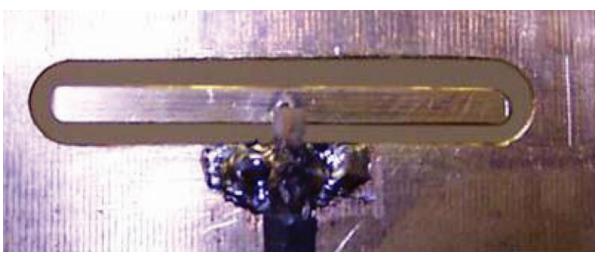

(b)

FIGURE 3: Folded slot forward and backward side.

TABLE 1: Slot antenna resonance frequency by changing the size of ground plane (measurement).

\begin{tabular}{lc}
\hline Size of ground plane & Resonance frequency $(\mathrm{GHz})$ \\
\hline $10 \lambda \times 10 \lambda$ & 4.585 \\
$5 \lambda \times 5 \lambda$ & 4.650 \\
$\lambda \times \lambda$ & 4.835 \\
$0.5 \lambda \times 0.5 \lambda$ & 4.325 \\
\hline
\end{tabular}

Using (1) the folded slot dipole (Figure 3) was chosen to the basic dipole element of the MIMO antenna because of its well-matched impedance to $50 \mathrm{ohm}$ coaxial transmission line without necessity of using any matching circuit.

As start the simulation and optimization of the simple folded slot dipole over plane ground conductor was made. The theoretical electrical vector field distribution and the simulated one can be seen in Figure 2.

After the first simulations and modeling, the slot antennas were designed using Ansoft HFSS electromagnetic simulator. Optimization was used to get matched input impedance at the desired working frequency. Finally the antennas are realized on copper plate with a thickness of $0.5 \mathrm{~mm}$, and the excitation is through $50 \mathrm{ohm}$ coaxial cables.

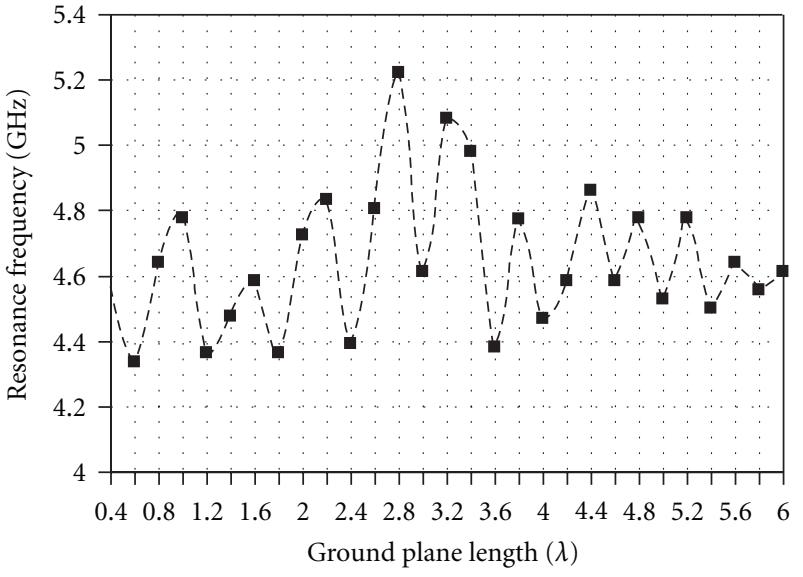

FIGURE 4: Resonance frequency of slot antenna versus ground plane size.

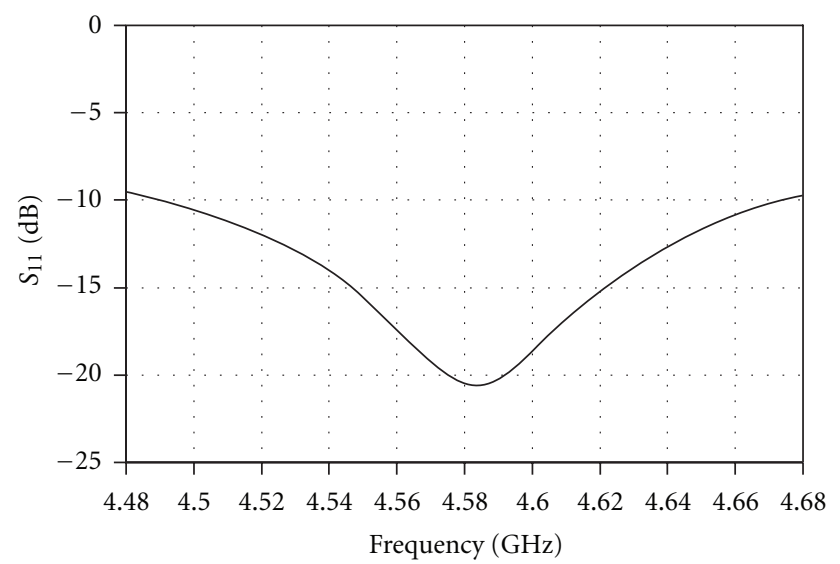

Figure 5: Measured input reflection of slot dipole over a $10 \lambda$ by $10 \lambda$ ground plane conductor.

The slot antenna over a notable size copper plate was realized and measured. The first results showed good agreement on HFSS-based simulations. In the simulation and at the realization size of $10 \lambda$ by $10 \lambda$ ground plane was used, but later at forming the MIMO cube the size of plane was reduced and the antenna resonance frequency remarkably changed (Figure 5).

The effect of rectangular ground plane size was also investigated on the resonance frequency by HFSS simulations as can be seen on Figure 4 .

Figure 4 shows a notable change of resonance frequency especially in the $2.4 \lambda$ length range and for feasible sizes notably decreases but at the design of MIMO antenna has to be taken into account (Table 1).

The investigated antenna elements provide almost lossless realization on cheap metallic plate. Two types of slots are simulated, optimized, and measured for $4.5-4.6 \mathrm{GHz}$. Coupling between dipoles is also measured and presented. The cube antennas exhibit an impedance bandwidth (VSWR $<2$ ) of $18 \%$ and $7 \%$. 


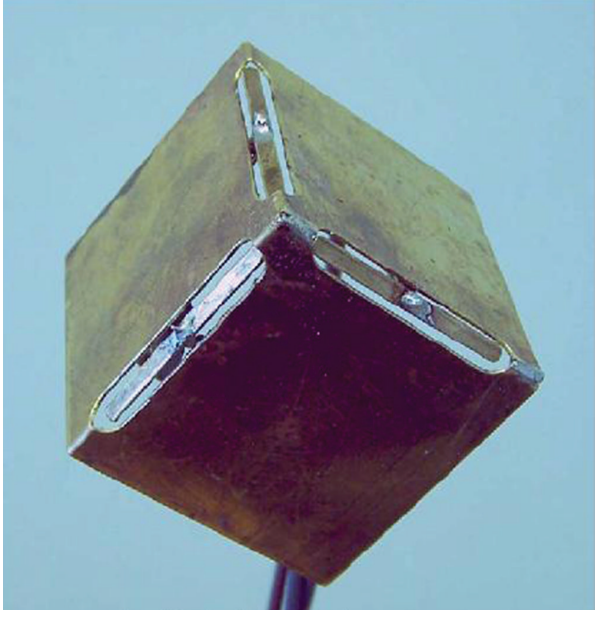

Figure 6: MIMO cube with slot dipoles on edges.

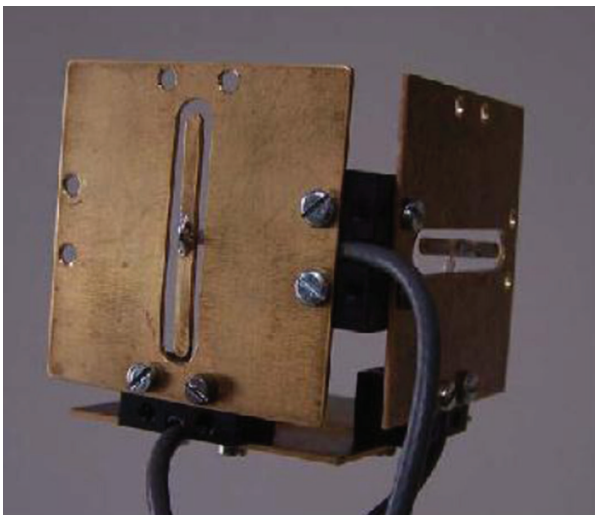

Figure 7: MIMO cube with slot dipoles on separated planes.

Results of simulations show that the theoretical capacity due to mutual coupling between MIMO antennas is lower than for implemented antennas with mutual coupling for cube side lengths less than about $0.3 \lambda$. For the prototype antenna, the $S$ parameter isolation between ports is below $-35 \mathrm{~dB}$ over a bandwidth of $300 \mathrm{MHz}$, and therefore excellent MIMO antenna can be formed without capacity degradation.

After the first tests two MIMO cube antennas have been analyzed and realized for the measurements and comparison of especially the mutual coupling between the antenna elements.

The first structure realized prototype is a part of a MIMO cube with slot dipole on the edges (Figure 6). The detailed analysis indicates that surface currents flow on the board, giving an isolation decrease. Our second structure applies therefore separated boards for decreasing the coupling (Figure 7).

For each antenna, the input reflection is acceptable for many mobile communications application over a bandwidth of at least $300 \mathrm{MHz}$. The coupling between slot dipoles contrarily is unacceptable for the first MIMO cube in Figure 8 without any compensation in comparison with Figure 9. The effect of coupling can cause MIMO capacity

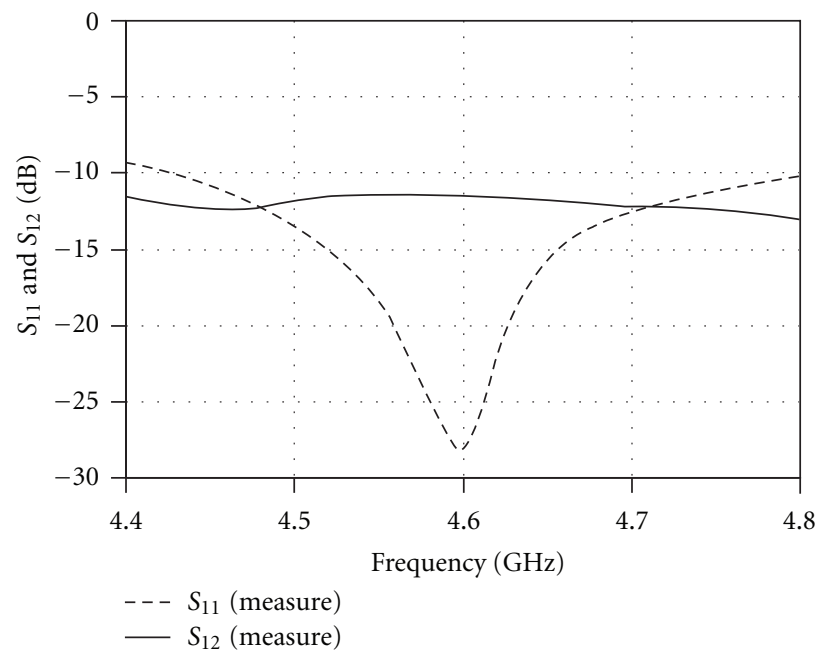

Figure 8: Measured MIMO antenna input reflection and coupling $\left(S_{11}\right.$ and $\left.S_{21}\right)$ for dipoles on edges.

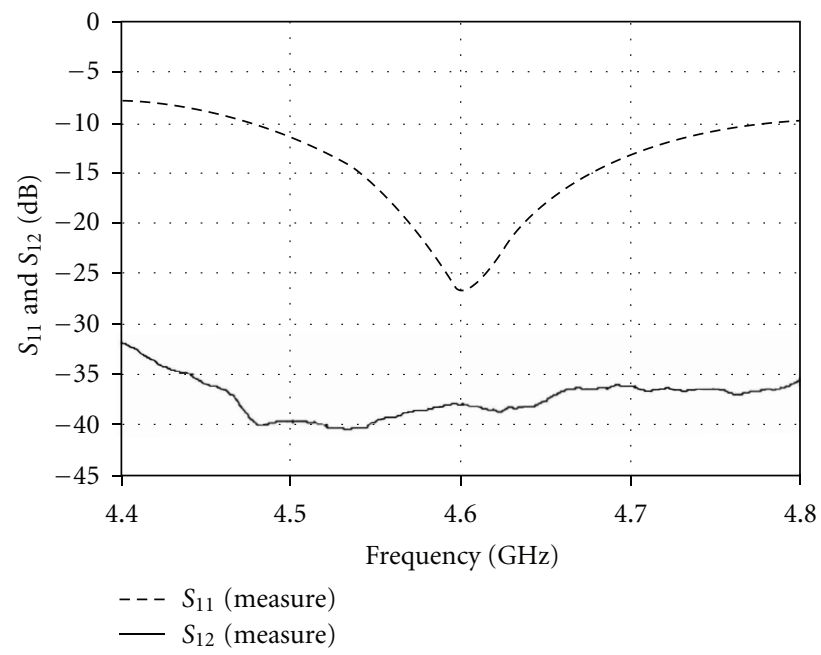

Figure 9: Measured MIMO antenna input reflection and coupling $\left(S_{11}\right.$ and $\left.S_{21}\right)$ for dipoles on separated planes.

degradation in real applications. The other effect on forming the MIMO cube from our antennas is the slight change in the resonance frequency which can be easily corrected by increasing the slot antenna length.

These first results enforced our farther investigation toward implementing optimization procedure which can take into account the mutual coupling effect for MIMO cubes and can be applied to capacity optimization. In the next part of the paper, the geometry optimization of MIMO antenna is shown for enhanced capacity.

\section{MIMO Antenna Simulation Model}

In our next simulation investigation, the MIMO system contains three wire dipole antennas both on the transmitter and the receiver sides. 


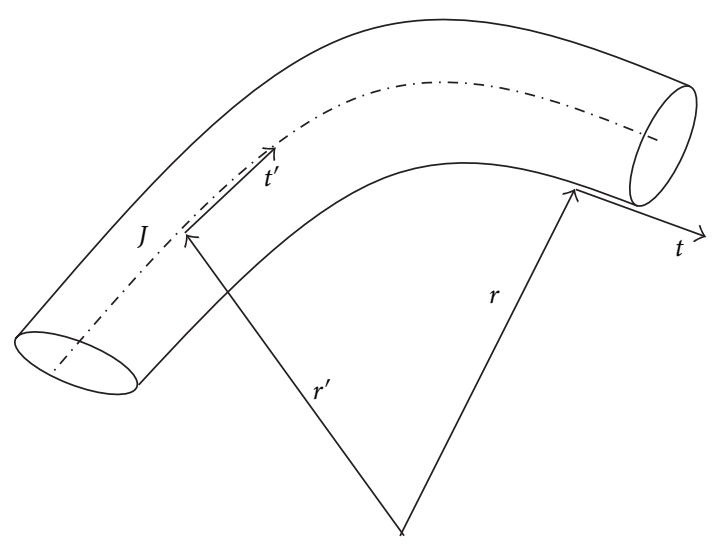

Figure 10: Thin wire geometry.

3.1. Wire Antenna Analysis. Let us consider an antenna consisting of many arbitrary-oriented wire elements. Starting with Maxwell equations and by enforcing the boundary condition for the total tangential electrical field on the antenna wire, it is possible to obtain the simplified general integral equation for arbitrary-oriented wires. Enforcing the boundary condition, the electrical field can be derived from the A magnetic vector potential.

Pocklington's procedure applied to our slot dual wire antennas supposes the current to be located over a thin filament over the conductor (Figure 10).

Pocklington's integral equation can be gotten finally:

$$
E_{\tan }^{i}=-\frac{1}{4 \pi j \omega \varepsilon} \int_{L}\left(t \cdot t^{\prime} k^{2} I \frac{e^{-j k R}}{R}+\frac{\partial}{\partial l^{\prime}} I \frac{\partial}{\partial l} \frac{e^{-j k R}}{R}\right) d l^{\prime}
$$

The solution of (2) should be determined using standard MM and Galerkin method. To solve (2) we used piecewise sinusoidal expansion and weighting functions.

The resulting mutual impedance between MIMO antenna elements can be obtained using the $N$ port analysis to the whole system of antennas.

At the optimization process described in Section 3.3 the angle between antennas is the optimization variable as we are searching for the maximum channel capacity. In our simple case only these angles of the orientation of antennas are changed, but the method introduced is applicable also for general antenna position optimization.

For a MIMO radio channel with channel matrix $H$, the $S V D$ is given as $\mathbf{H}=S V D^{T}$, where $S$ and $D^{H}$ (complex conjugate transpose of $D$ ) are complex unitary matrices, $V=$ $\operatorname{diag}\left(\sqrt{\lambda_{1}}, \ldots, \sqrt{\lambda_{r}}\right)$ diagonal square matrix with $\lambda_{1}, \lambda_{2}, \ldots, \lambda_{r}$ being the positive eigenvalues of $\mathbf{H H}^{\mathrm{H}}$, and $r \leq \min \{M t, N r\}$ denotes the rank of $\mathbf{H H}^{\mathrm{H}}$. With the assumption of known channel at the transmitter, the theoretical capacity from water filling is given as

$$
C=\sum_{i=1}^{r^{\prime}} \log _{2}\left(1+\lambda_{i} \mathrm{SNR}_{i}\right)
$$

where $\mathrm{SNR}_{i}=P_{t} / \sigma^{2}$ is the individual SNR of the eigenmodes after water filling and $r^{\prime}$ denotes the number of useful eigenmodes with positive power allocation.

In the course of calculation, the channel matrix is normalized with the average path gain $\Psi$ :

$$
\Psi^{2}=\left\{\frac{1}{m_{\mathrm{tr}} m_{\mathrm{re}}} \sum_{i=1}^{m_{\mathrm{tr}}} \sum_{j=1}^{m_{\mathrm{re}}}|H(i, j)|^{2}\right\},
$$

where $m_{\mathrm{rt}}$ and $m_{\mathrm{re}}$ are the numbers of antennas on both sides. For computing the average path gain it shall make the expectation value for all random channels.

3.2. 3D-Environment Simulation Model. The antenna system is situated in a $3 \mathrm{D}$ scattering environment indoor channel. Waves of arbitrary polarizations are incident on the antenna structure from all possible directions. The waves launch from the transmitter antennas, and first they reach the elements of the primary reflection surface, and from here they rescatter to the second group of scatterers, and finally they are reflected to the receiving antennas. The transmission matrix $(\mathbf{H})$ which connects the receiver and transmitter antennas is filled by assuming DB scattering $[13,14]$.

The scatterers have a random scattering polarization matrix, and they are positioned randomly and uniformly on a ball-shaped surface around the transmitter and the receiver antennas.

$\mathbf{H}$ matrix of the DB model consists of three differentsized submatrices:

$\mathbf{H}_{\text {tr-scl }}$ means the transfer between the transmitter unit and the first surface of scatterers;

$\mathbf{H}_{\text {sc1-sc2 }}$ is the connection between the first group and the second group of scatterers;

$\mathbf{H}_{\text {sc2-re }}$ is like the first matrix that can describe the path from the second group of scatterers to the receiver.

We chose the number of scatterer points on the reflection surface to be hundred. The transmission matrices $\mathbf{H}_{\mathrm{trsc}}$, $\mathbf{H}_{\text {sc1-sc2 }}$, and $\mathbf{H}_{\text {sc2-re }}$ are of sizes $\mathrm{Sm} \times \mathrm{Mt}, \mathrm{Sn} \times \mathrm{Sm}$, and $\mathrm{Nr} \times$ $\mathrm{Sn}$. Therefore, the complete MIMO transmission matrix, $\mathrm{H}$, may be given as

$$
\mathbf{H}=\mathbf{H}_{\mathrm{sc} 2-\mathrm{re}} \cdot \mathbf{H}_{\mathrm{sc1}-\mathrm{sc} 2} \cdot \mathbf{H}_{\mathrm{sc} 1-\mathrm{tr}},
$$

where $\mathbf{H}$ is of size $\mathrm{Nr} \times \mathrm{Mt}$ (in this case the element number of $H$ is 9) with entries $\mathbf{H}[n, m]$ which describe the path from $n_{\text {th }}$ receiver to $m_{\text {th }}$ transmitter.

Each entry of the transmission matrices is computed using (5) for every radiation direction pointing either to or from the scatterers. The received incident electric field at the elements of the first reflection surface (at the first scatter points) around the transmitter unit has $\theta$ and $\varphi$ direction components:

$$
\begin{aligned}
& \mathrm{H}_{\text {scl-re }, \vartheta}=E_{\text {rad }, \vartheta}, \\
& \mathrm{H}_{\text {scl-re }, \varphi}=E_{\text {rad }, \varphi .} .
\end{aligned}
$$




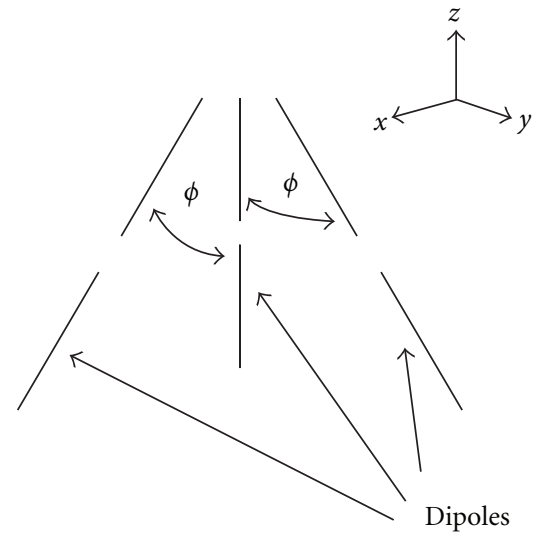

(a)

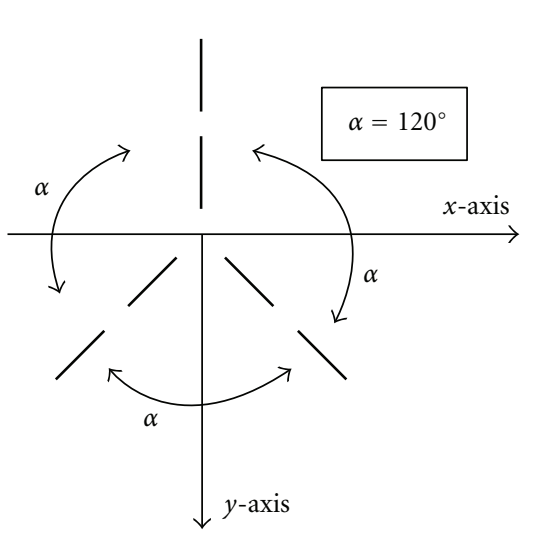

(b)

FIGURE 11: The $3 \times 3$ MIMO antenna structure for maximizing the mean capacity by rotation of the antennas, parallel at the transmitter and the receiver units, from the $z$-axis toward $x-y$ plane (it is opened like an umbrella).

The connection between the first and the second scatterers is described in the $\mathbf{H}_{\text {sc1-sc2 }}$, which is a $162 \times 162$ square matrix where its entries are random complex numbers with Gaussian distribution. In this case the incident signal at the element of the second scattering surface is given by

$$
\begin{aligned}
& E_{r, 9}=\mathrm{H}_{\mathrm{scl}-\mathrm{sc} 2} \cdot \mathrm{H}_{\mathrm{scl} 1-\mathrm{re}, 9}, \\
& E_{r, \varphi}=\mathrm{H}_{\mathrm{scl}-\mathrm{sc} 2} \cdot \mathrm{H}_{\mathrm{scl}-\mathrm{re}, \varphi} \cdot
\end{aligned}
$$

The transmission matrix, $\mathrm{H}$, is computed from the induced voltage on the receiving antenna elements. If there are $W$ multipath incidents on the receiving antenna, the induced voltages from the direction $\vartheta$ and $\varphi$ are given as

$$
\begin{aligned}
V_{i, \vartheta} & =\sum_{w=1}^{W} E_{r, \vartheta, w} \cdot h_{\mathrm{eff}, \vartheta, w}, \\
V_{i, \varphi} & =\sum_{w=1}^{W} E_{r, \varphi, w} \cdot h_{\mathrm{eff}, \varphi, w},
\end{aligned}
$$

where $h_{\text {eff }}$ is the vector effective length of the dipole which is given as $h_{\text {eff }}=\left(E_{\mathrm{rad}} / j \beta I_{\mathrm{in}} \eta\right) \cdot 4 \pi r e^{j \beta r}$. Here $I_{\text {in }}$ is the excitation.

Our multiple-antenna system is composed of $M_{t}=3$ and $N_{r}=3$ electric dipoles at both the transmitter and the receiver units. In this way, the transmission channel matrix $\mathrm{H}$ consists of nine transmission links $(3 \times 3)$. At the start of the simulation the antennas were orientated in the $z$-axis and later they were rotated toward the $x-y$ axes (the structure was opened like an umbrella). The radiated electric field of each dipole is applied for the calculation of the transmitter matrix. The current distribution for each electric dipole is sinusoidal, which is often supposed for finite-length dipoles. Figure 11 shows the method of rotating of antennas in the simulation structure.

This simulation model statistically describes the material, surface, and motion of these objects which results in phase and amplitude error in the course of propagation. By this method we could describe the continuously varying indoor environment.

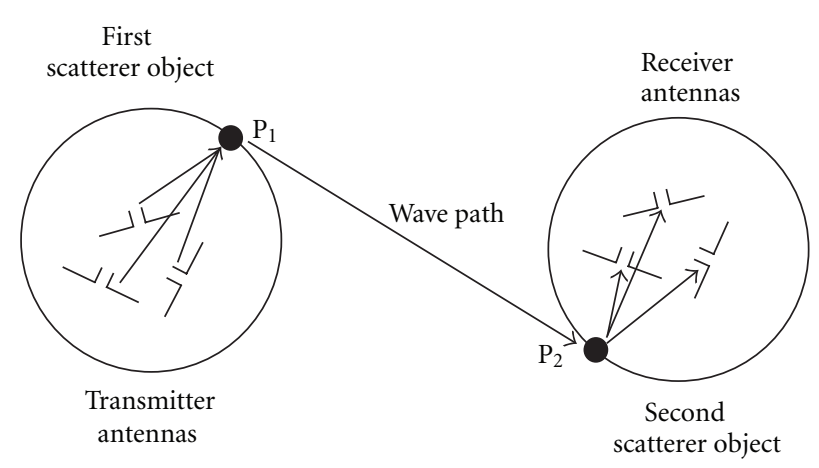

FIGURE 12: Signal wave path in a scattering environment. Transmitter and receiver dipole antennas in a DB scattering environment.

3.3. Genetic Algorithm-Based MIMO Antenna Orientation Optimization. The MIMO capacity enhancement calculation can be performed already by using the previously summarized mathematics, but because of the remarkable computational demand we do have test by using Genetic Algorithm- (GA-) based calculations for capacity maximization. In that case the antenna orientations on transmit and receive sides are coded for the GA in the region of angle $0 \ldots 120$ degree (Figure 13).

Genetic Algorithms are increasingly being applied to difficult global optimization problems. GA optimizers are robust, stochastic search methods modeled on the principles and concepts of natural selection $[10,11]$.

If the transmit and receive antenna orientation is fully described by $N_{\text {par }}$ parameters arranged in a vector $x=$ $\left\{x_{i} \mid i=1, \ldots, N_{\mathrm{par}}\right\}$ that is considered, then the knowledge of $x$ permits the evaluation of the objective function $f(x)$, which indicates the worth of a design (the area coverage percentage). It is assumed that $x_{i}$ takes on either real or discrete values and that $f(x)$ needs to be maximized.

In our approach in $x$ we coded the $\phi$ angles and the objective function $f(x)$ is the channel mean capacity. 


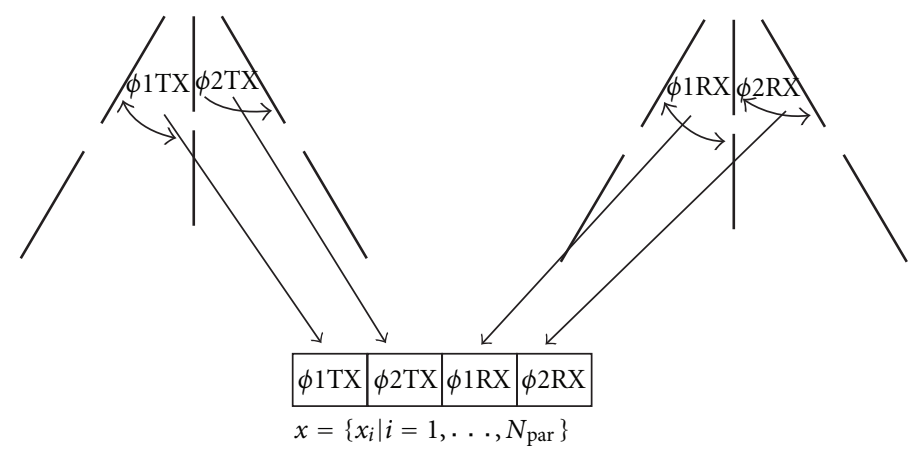

Figure 13: Coding scheme for GA.

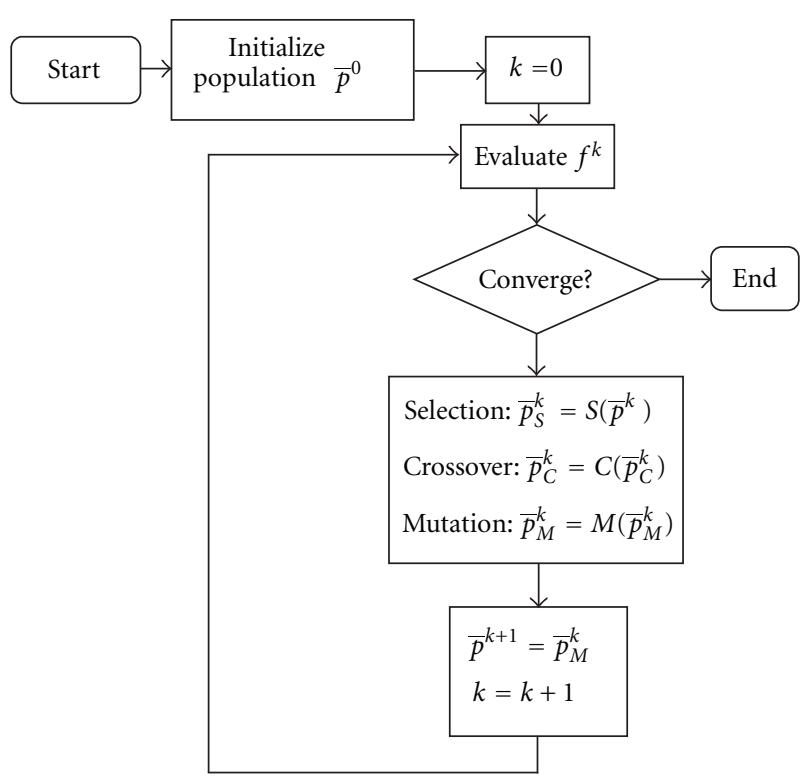

FIGURE 14: Simple GA flowchart.

The GA (Figure 14) does not operate on $x$ but on a discrete representation or chromosome $p=\left\{g_{i} \mid i=\right.$ $1, \ldots, N\}$ of $x$, each parameter $x_{i}$ being described by a gene $g_{i}$. Each gene $g_{i}$ in turn consists of a set of $N_{\text {all }}^{i}$ all that are selected from a finite alphabet and that together decode a unique $x_{i}$.

The GAs do not limit themselves to the iterative refinement of a single coded design candidate; instead the simple GA (SGA) simultaneously acts upon a set of candidates or population

$$
\bar{p}=\left\{p(i) \mid i=1, \ldots, N_{\text {pop }}\right\}
$$

where $N_{\text {pop }}$ is the population size.

Starting from an initial population $\bar{p}^{0}$, the SGA iteratively constructs populations $\bar{p}^{k}, k=1, \ldots, N_{\text {gen }}$, with $N_{\text {gen }}$ denoting the total number of SGA generations. Subsequent generations are constructed by iteratively acting upon $\bar{p}^{0}$ with a set of genetic operators. The operators that induce the transition $\bar{p}^{k} \rightarrow \bar{p}^{k+1}$ are guided solely by knowledge of the vector of objective function values

$$
f^{k}=\left\{f\left(x\left(p^{k}(i)\right)\right) \mid i=1, \ldots, N_{\text {pop }}\right\}
$$

and induce changes in the genetic makeup of the population leading to a $\bar{p}^{k+1}$ comprising individuals that are, on average, better adapted to their environment than those in $\bar{p}^{k}$, that is, they are characterized by higher objective function values.

This change is affected by three operators mentioned in the introduction: selection $(S)$, crossover $(C)$, and mutation $(M)$.

The selection operator implements the principle of survival of the fittest. Acting on $\bar{p}^{k}, S$ produces a new population $\bar{p}_{S}^{k}=S\left(\bar{p}^{k}\right)$ again of size $N_{\text {pop }}$ that is, on average, populated by the better-fit individuals present in $\bar{p}^{k}$. Among the many existing schemes, tournament selection has been chosen. The crossover operator mimics natural procreation. Specifically, $C$ acts upon the population $\bar{p}_{S}^{k}$ by mating its members, thereby creating a new population

$$
\bar{p}_{C}^{k}=\bigcup_{i=1}^{N_{\text {pop }} / 2} C\left(\operatorname{ch}\left(\bar{p}_{S}^{k}\right), \operatorname{ch}\left(\bar{p}_{S}^{k}\right)\right),
$$

where the chromosome crossover operator $C$ selects a random crossover allele $a_{N \text { cross }}$ between the two chromosomes to be crossed upon which it acts with probability $P_{\text {cross }}$.

The mutation operator generates a new population of size

$$
\bar{p}_{M}^{k}=\bigcup_{i=1}^{N_{\text {pop }}} M\left(\bar{p}_{C}^{k}(i)\right) .
$$

by introducing small random changes into $\bar{p}_{C}^{k}$. The action of $M$ can be represented in operator form as

$$
\bar{p}_{M}^{k}=\bigcup_{i=1}^{N_{\text {pop }}} M\left(\bar{p}_{C}^{k}(i)\right) .
$$

The cost function of the optimization procedure has been the channel mean capacity, $\phi$ is the angle between antennas:

$\cos t=C(\phi)$. 
TABLE 2: Simulation time for GA at different probability parameters (mutual coupling and conjugate antenna matching).

\begin{tabular}{lcc}
\hline Mutation probability & Crossover probability & Optimization time \\
\hline $0.5 \%$ & $1 \%$ & $57 \mathrm{~min}$ \\
$1 \%$ & $1 \%$ & $54 \mathrm{~min}$ \\
$1 \%$ & $2 \%$ & $26 \mathrm{~min}$ \\
$1 \%$ & $5 \%$ & $120 \mathrm{~min}$ \\
\hline
\end{tabular}

The effect of proper choose of the Crossover and Mutation probabilities on duration of optimization are illustrated in Table 2.

\section{Simulation Results}

4.1. Effect of Mutual Coupling for the Channel Capacity. The GA optimization procedure has been used for three cases without taking into account the antenna mutual couplings, with antenna mutual couplings using conjugate matching and with $50 \mathrm{ohms}$ terminations on receiver antennas. The optimization times depend significantly on the GA parameters like mutation and crossover probabilities. In the simulation, 200 random scatterers have been used on each bounce, the angle resolution for antenna orientation $2^{\circ}$.

The GA gives the optimum for antenna orientation, but for comparing the three cases a full search has been made with an orientation angle resolution of $10^{\circ}$ and the same orientation angles are used symmetrically for the transmit and receive antennas. We simulated the motion of the antennas by the above-described rotation method. First of all in this simulation the antennas are parallel to the $z$-axis. In the midst of the simulation the antennas opened in the space like an umbrella. In the end of the simulation the antennas reached the $x-y$ plane. In this case the antennas are on the farthest position, which by the phase between antenna and $z$ axis was changed from $0^{\circ}$ to $90^{\circ}$. The result of the simulation shows perfect symmetry for the $x-y$ plane. We look for the perfect position for the maximal mean channel capacity in consideration of the effect of mutual coupling in case of conjugate matching and nonconjugate matching. Figure 15 shows the mean capacity versus the angle of rotation. The simulation parameters are $S / N=20 \mathrm{~dB}$, and length of antennas are $0.45 \lambda$.

In the case of neglected antenna coupling, the maximal channel capacity is at about $\phi=45^{\circ}$ antenna angle. In case of conjugate matching, the maximal channel capacity is at $70^{\circ}$ and without conjugate matching the capacity is maximal and approximately constant from $50^{\circ}$.

Finally we chose the $90^{\circ}$ structure, as a matter of fact the MIMO cube, because the realization easiness and the capacity increase in the angle region of $40-90^{\circ}$ is not really considerable. The implemented GA algorithm later will be used for the general position optimization of the MIMO antennas, and with this increase of freedom we expect a much higher capacity gain and a more compact structure than the MIMO cube.

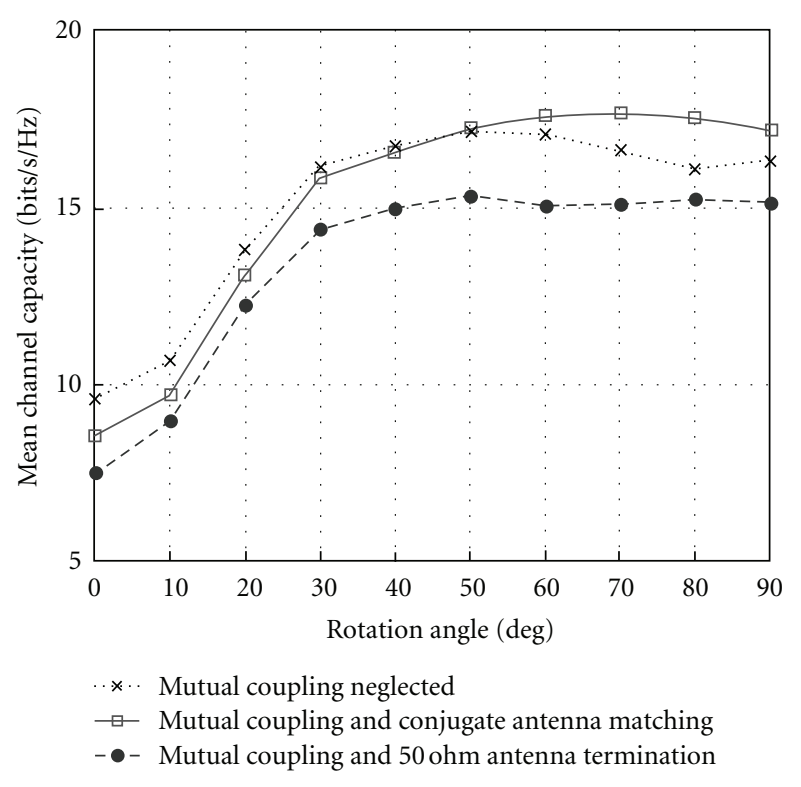

Figure 15: Channel capacity of a $3 \times 3$ MIMO antenna system, in case of neglected antenna coupling, mutual coupling with and without conjugate matching.

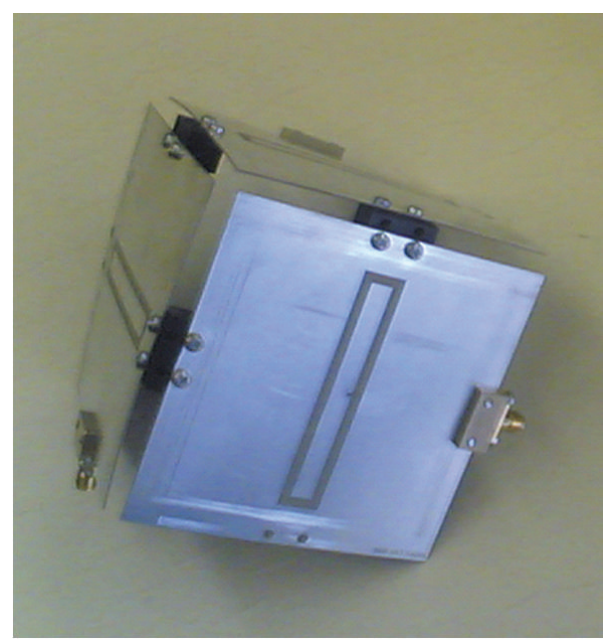

Figure 16: 3 antennas on the mid of the faces.

\section{Final Measurement}

Figures 16 and 18 show the realized antenna structures. The measurement results are the $S_{i j}$ (mutual coupling) and $S_{i i}$ (reflection) parameters of the antennas. Figures 17 (antennas on faces) and Figure 19 (antennas on edges) show these results.

The measurements setup is part of an existing receiver beamforming array, which has the main characteristics of operation in $2 \mathrm{GHz}$ band, 4 parallel $I-Q$ receiver channel, $-102 \mathrm{dBm}$ receiver sensitivity for $3 \mathrm{~dB}$ SNR, 12 bits ADC, real-time sampling on every channel simultaneously, fast inbuilt DSP for embedded signal processing (380 MIPS). The system control is on USB interface using LabVIEW control and processing software. Our measurement system contains 


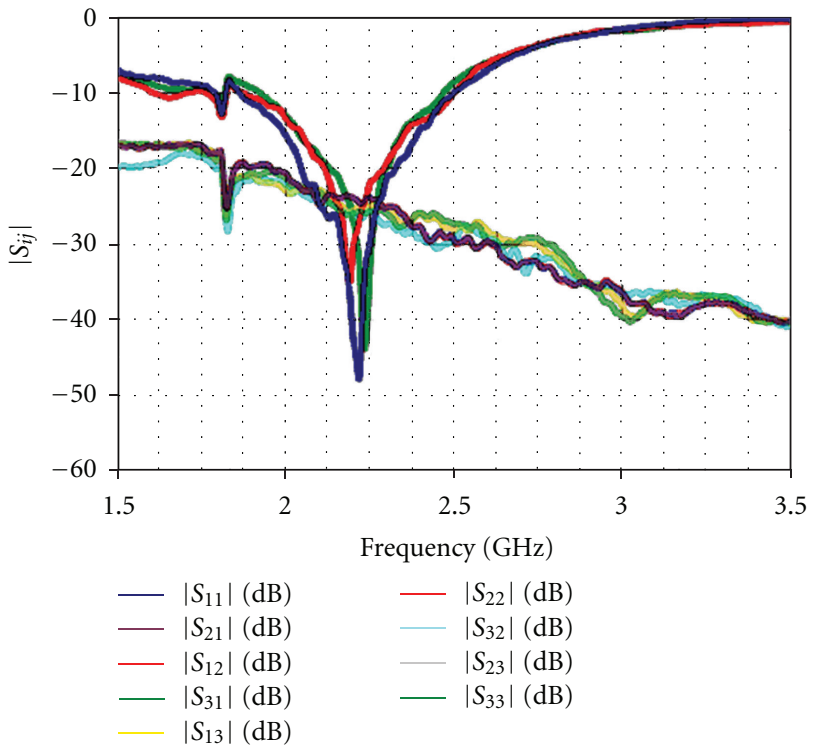

FIGURE 17: 3 antennas on the mid of the faces-measured $S$ parameters.

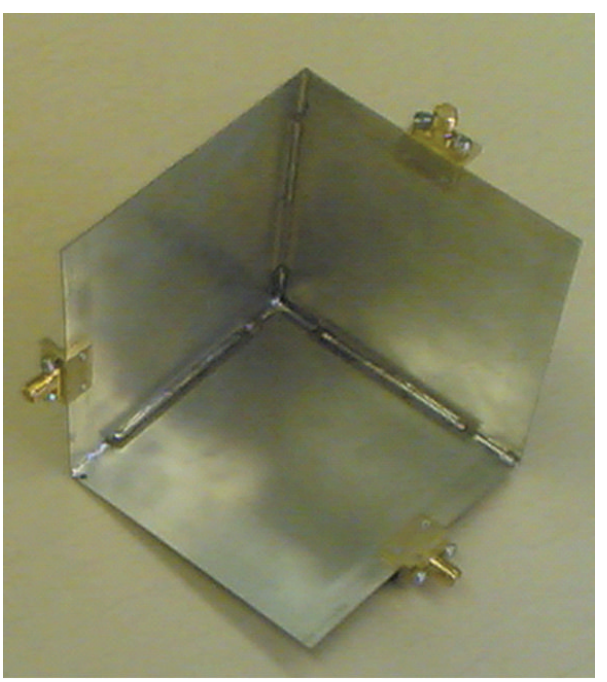

FIGURE 18: 3 antennas on the edges.

three major parts: the receiver blocks, the DSP unit, and the measuring software running on a laptop computer. The four receiver units with overall 4 input channels operate at 2.2 GHz center frequency, after double conversion baseband $I-Q$ signals are sampled and converted to 12 bit data. The DSP can provide sampling numbers having the powers of $2(1,2,4, \ldots, 256)$. The receiver antennas are the newly developed MIMO antennas. For our measuring setup only three-channel receiver was used from the existing 4 because of the MIMO antenna which has 3 outputs. On the transmit side, the same MIMO antenna has been used but only SIMO measurement has been performed with our single transmitter.

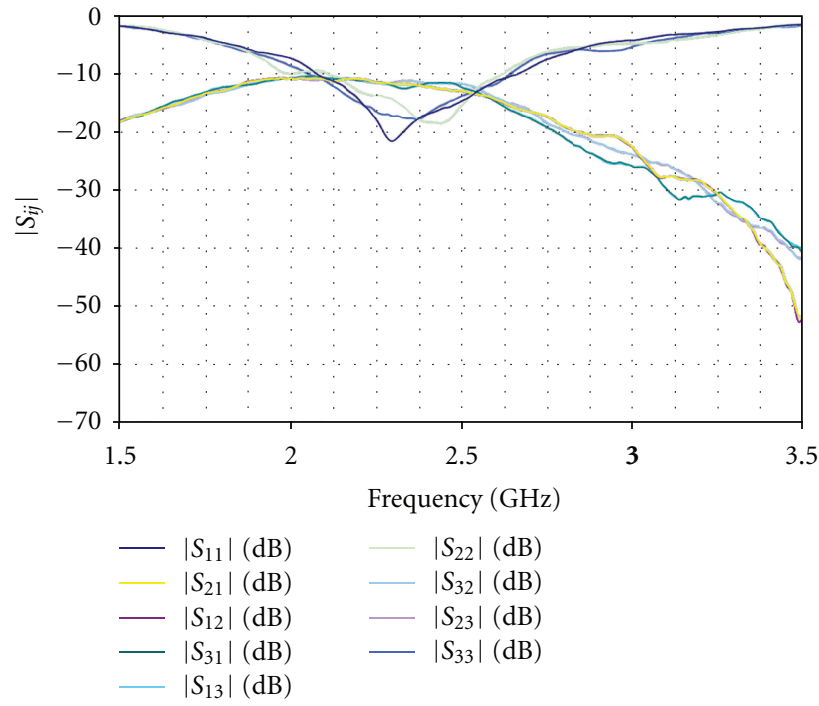

Figure 19: 3 antennas on the edges-measured $S$ parameters.

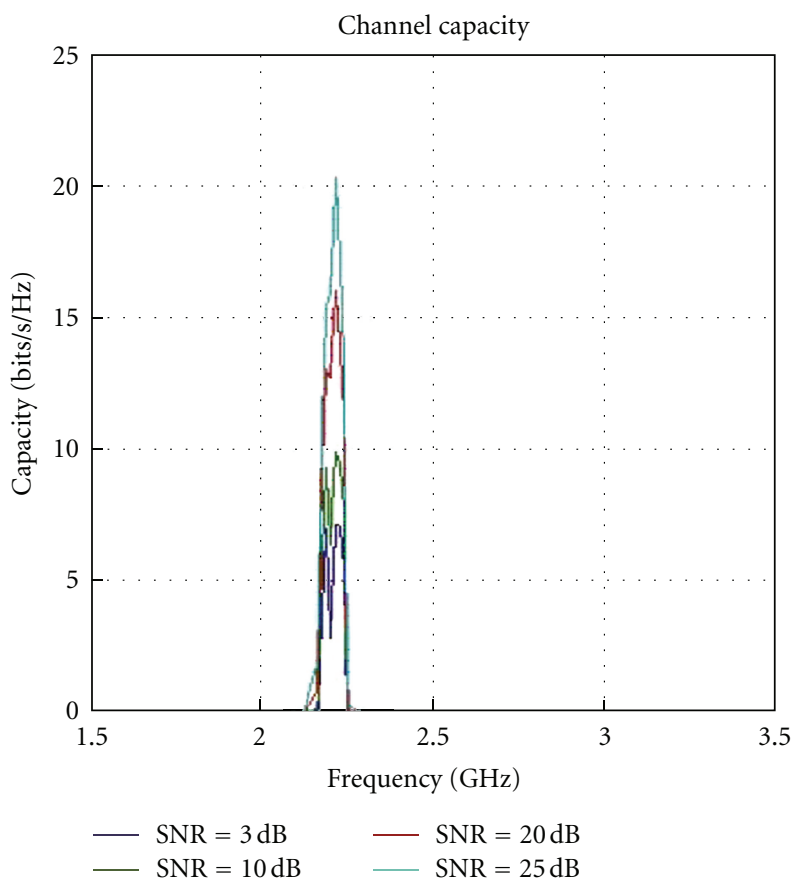

FIGURE 20: 3 antennas on the mid of the faces-calculated channel capacity (from channel transfer matrix).

Based on the three independent SIMO measurements, we got the channel transfer matrices and calculated the channel capacity using (3).

The results (Figures 20 and 21) show that the first structure (antennas on the faces) realizes higher channel capacity than the second one with antennas on edges, but this maximal capacity can be gotten only in a very narrow bandwidth; therefore, for the present wideband wireless data applications, the edge placed slots give and enhanced capacity. 


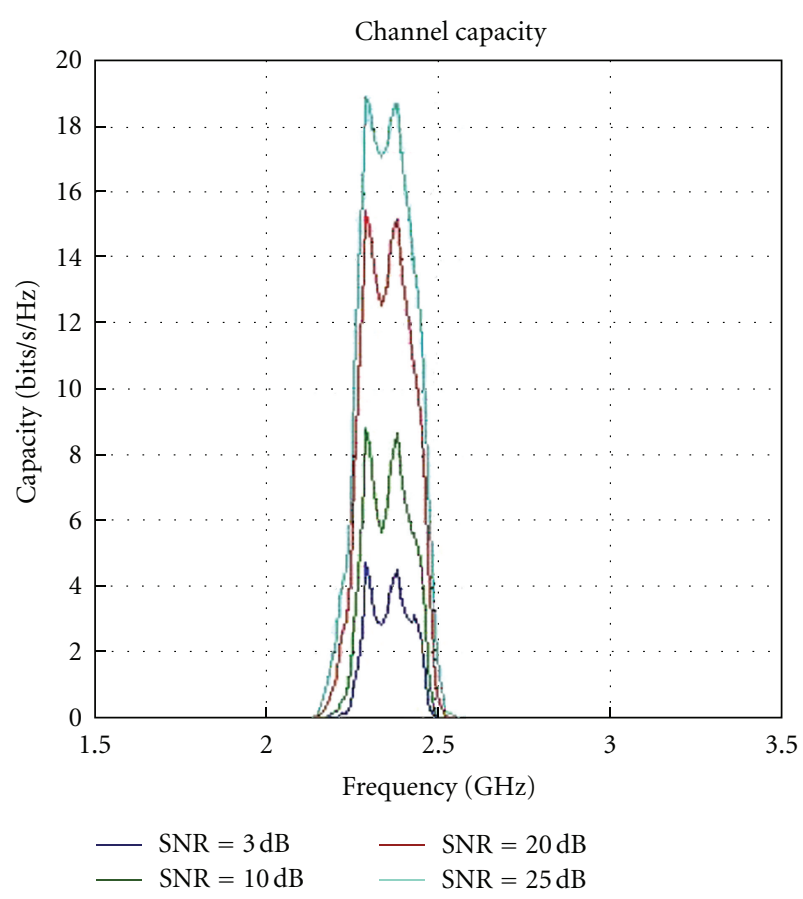

FIGURE 21: 3 antennas on the edges-calculated channel capacity (from channel transfer matrix).

\section{Conclusion}

In this paper, we investigated a $3 \times 3 \mathrm{MIMO}$ antennas system. We made simulations for analysis of the effects of antenna positions for the mean channel capacity. We found that the maximal channel mean capacity is affected by structure in which the antennas are perpendicular to each other. With this we examined the structure with conjugate and nonconjugate matching. Based on the result of the simulation in the realized and measured structure, the antennas were perpendicular to each other. The measurements confirm our results of simulations.

The next step in our research is developing the full MIMO transmitter-receiver measurement system, with which direct capacity measurements can be fulfilled.

\section{Acknowledgments}

The research work leading to this paper has been supported by the European Commission under SAMURAI. This work is connected to the scientific program of the "Development of quality-oriented and harmonized $\mathrm{R}+\mathrm{D}+\mathrm{I}$ strategy and functional model at BME" project. This project is supported by the New Széchenyi Plan (Project ID: TÁMOP-4.2.1/B09/1/KMR-2010-0002).

\section{References}

[1] C. Y. Chiu and R. D. Murch, "Design of a 24-port MIMO cube," in IEEE Antennas and Propagation Society, AP-S International Symposium, pp. 2397-2400, Honolulu, Hawaii, USA, June 2007.
[2] C. Y. Chiu, J. B. Yan, and R. D. Murch, "24-port and 36-port antenna cubes suitable for MIMO wireless communications," IEEE Transactions on Antennas and Propagation, vol. 56, no. 4, pp. 1170-1176, 2008.

[3] Á. Németh, L. Szücs, and L. Nagy, "MIMO cube formed of slot dipoles," in Proceedings of the 16th IST Mobile and Wireless Communications Summit, Budapest, Hungary, July 2007.

[4] N. Behdad, "A uniplanar dual-polarized miniaturized antenna," in Proceedings of the General Assembly and Scientific Symposium of the International Union of Radio Science (URSI '08), Chicago, Ill, USA, August 2008.

[5] A. Suyama, D. Uchida, H. Arai, Y. Inoue, and K. Cho, "A dual polarization 8-port MIMO antenna with dipole and slot antennas," IEICE Technical Report, Macao University, Macao, China, 2009.

[6] D. Uchida, H. Arai, Y. Inoue, and K. Cho, "A low-profile dual-polarized directional antenna for enhancing channel capacity in indoor MIMO systems," IEICE Transactions on Communications, vol. 93, no. 10, pp. 2570-2577, 2010.

[7] J. X. Yun and R. G. Vaughan, "Slot MIMO cube," in Proceedings of the IEEE International Symposium on Antennas and Propagation and CNC-USNC/URSI Radio Science Meeting (APSURSI '10), Toronto, Canada, July 2010.

[8] T. Svantesson and A. Ranheim, "Mutual coupling effects on the capacity of multielement antenna systems," in Proceedings of the IEEE Interntional Conference on Acoustics, Speech, and Signal Processing, vol. 4, pp. 2485-2488, Salt Lake City, Utah, USA, May 2001.

[9] R. Janaswamy, "Effect of element mutual coupling on the capacity of fixed length linear arrays," IEEE Antennas and Wireless Propagation Letters, vol. 1, pp. 157-160, 2002.

[10] Z. Michalewicz, Genetic Algorithms + Data Structures = Evolution Programs, Springer, Berlin, Germany, 1996.

[11] E. Michielssen, Y. Rahmat-Samii, and D. S. Weile, Electromagnetic System Design Using Genetic Algorithms, Modern Radio Science, 1999.

[12] L. Nagy, "Indoor radio coverage optimization for WLAN," in Proceedings of the 2nd European Conference on Antennas and Propagation (EuCAP '07), no. 11961, Edinburgh, UK, November 2007.

[13] B. N. Getu and J. B. Andersen, "The MIMO cube-a compact MIMO antenna," IEEE Transactions on Wireless Communications, vol. 4, no. 3, pp. 1136-1141, 2005.

[14] B. N. Getu and R. Janaswamy, "The effect of mutual coupling on the capacity of the MIMO cube," IEEE Antennas and Wireless Propagation Letters, vol. 4, no. 1, pp. 240-244, 2005. 

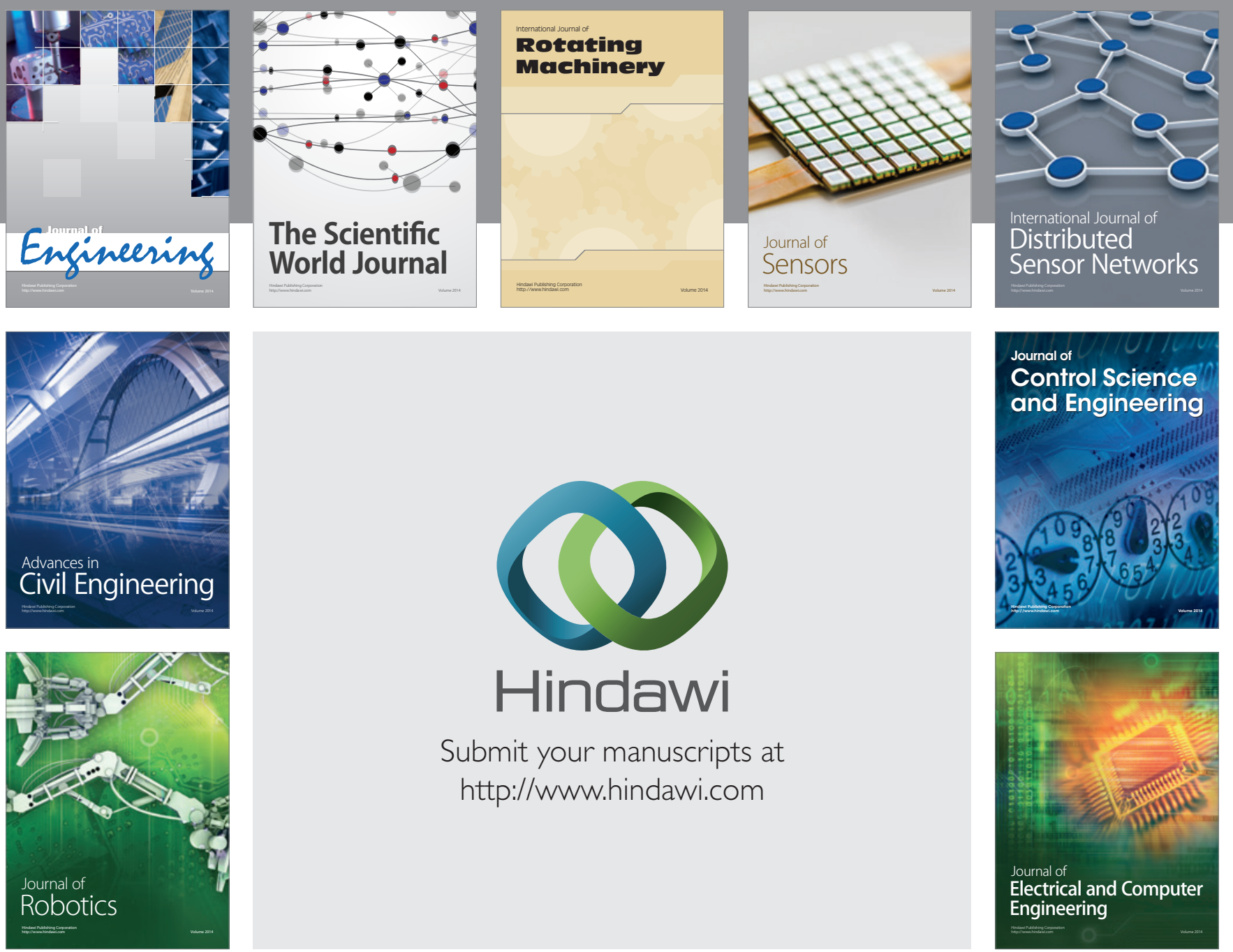

Submit your manuscripts at

http://www.hindawi.com
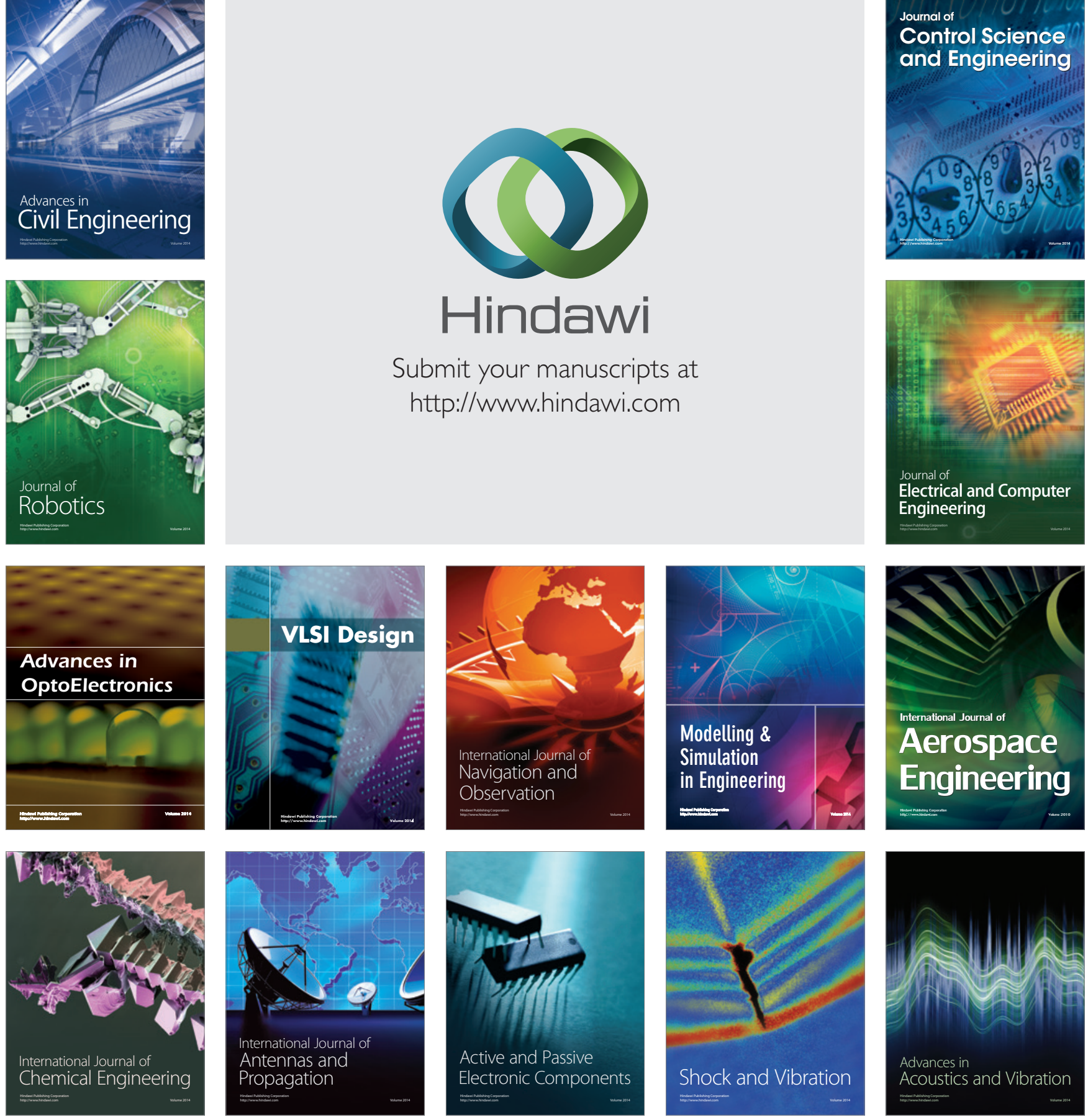